\title{
DELTA-SUNSPOTS AND X CLASS FLARES IN SOLAR CYCLE 22
}

\author{
ZHONGXIAN SHI and JINGXIU WANG \\ Beijing Astronomical Observatory, Chinese Academy of Sciences
}

\begin{abstract}
A sample of 221 active regions with $\delta$ magnetic configurations from 1986 to 1991 in Solar Cycle 22 is used to investigate the characteristics of $\delta$-sunspots and the relationship between the $\delta$-sunspots and $\mathrm{X}$ class $\mathrm{X}$-ray flares.
\end{abstract}

\section{FORMATION AND DISINTEGRATION}

It is well known that $\delta$-sunspots are highly productive of flares. All of the 132 $\mathrm{X}$ class X-ray flares in this solar cycle (before Dec.31, 1991) appeared in 56 active regions which presented $\delta$ magnetic configurations at some stage of their evolution. Therefore, to study the formation, disintegration, and other statistical properties of $\delta$-sunspot is of importance.

Many examples show that individual dipoles tend to expand independently with different orientation and separation speed. For some favorable distributions, a leading polarity from one dipole pushes into the following polarity of another dipole, or vice verse. The interpenetration, or in other words, the collisionmerging of opposite polarities eventually results in the formation of a $\delta$-sunspot. Further proper motions of those individual dipoles, sometimes, even separate a $\delta$-sunspot into two pieces.

Based on the sunspot data of this solar cycle, particularly the Huairou vector magnetograms, we have confirmed the discovery made by Tang (1983), Zirin and Liggett (1987) that $\delta$-sunspots form from opposite polarity sunspots which come from different dipoles. As a new revelation, we find examples that proper motions of individual dipoles may cause the separation of opposite polarity sunspots which once locked as an unity of $\delta$-sunspot. This is contradictory with the conclusion of Zirin (1988) that true $\delta$-sunspots have never been seen to break apart.

An example of a $\delta$-sunspot separated by proper motions of individual dipoles is shown in Figure 1 by vector magnetograms, in which the transverse field is presented by short line segments with length proportional to the field strength and alignments parallel to the direction, the line-of-sight field is presented by isogauss contours with the solid (dashed) lines for positive (negative) polarity. The isogauss levels are $\pm 40,80,160,320,640,1280,1600, \ldots$ gauss.

On Oct.19, sunspots F2, F2', and P1 are locked as a $\delta$-sunspot. The strong sheared transverse field is clearly seen on the neutral line. A total of 114 flares take place in this active region. However, F2, F2', and P1 all have different proper motions. On Oct.21, P1 and F2 had already separated from one another, they are no longer a $\delta$-sunspot. 


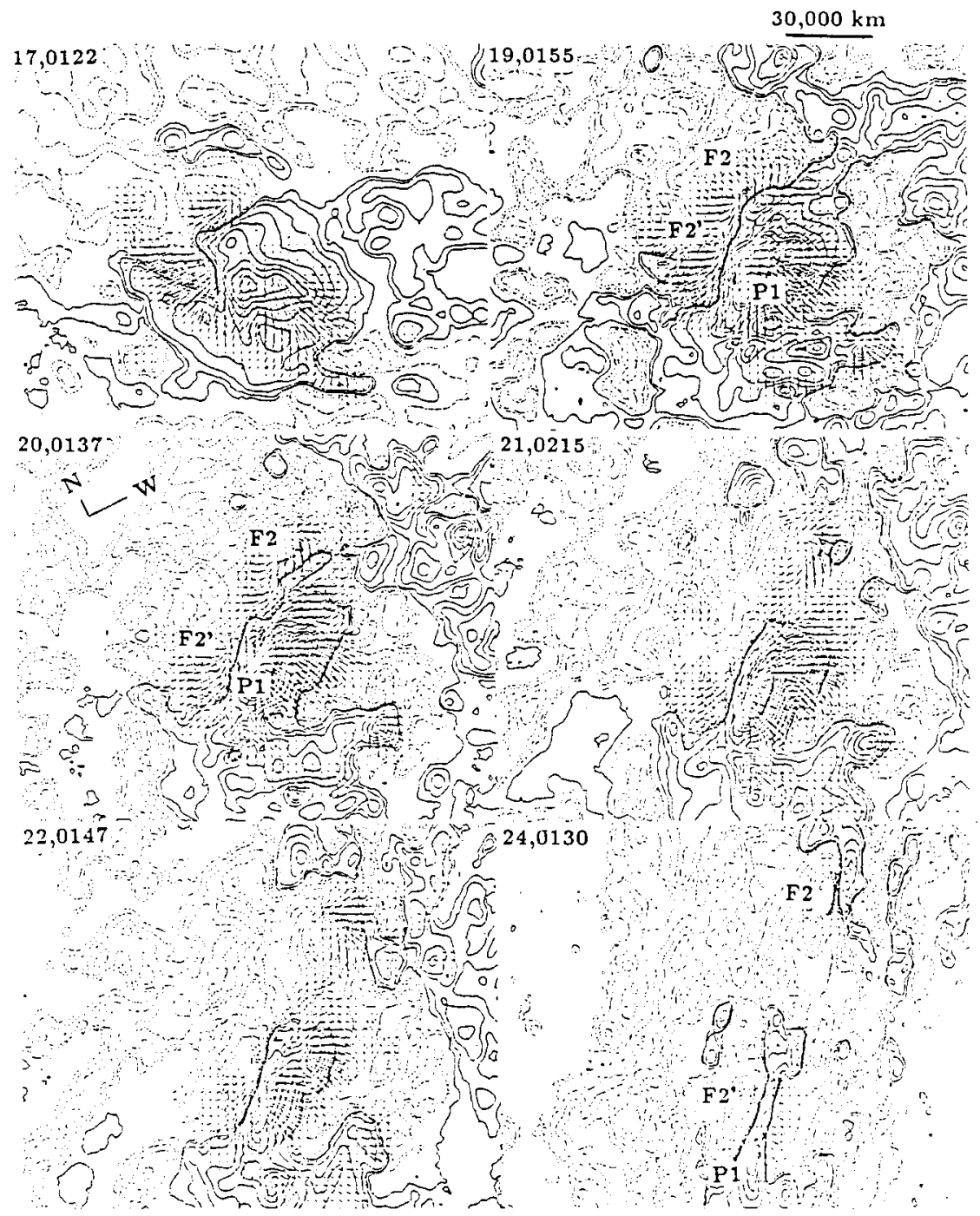

Fig.1 Time sequence of vector magnetograms showing the separation of a $\delta$-sunspot

\section{IMBALANCE OF MAGNETIC FLUX IN OPPOSITE POLARITIES}

It is known that active regions often have an imbalanced flux between leading and following polarities. The imbalance of magnetic flux in a $\delta$-sunspot appears more severe.

As the measurements of magnetic strength for sunspot umbra suffer from large errors caused by stray light, the estimation of magnetic flux is mainly based 
on sunspot area, as has been done by Sheeley (1966).

It seems that the $\delta$-sunspots which are highly productive of flares often have a serious flux imbalanc between opposite polarities. In this study, $35 \delta$ sunspots, which produced $104 \mathrm{X}$ class X-ray flares, with available sunspot data and magnetograms are chosen as a sample. The scatter plot of flux ratio of majority to minority polarity vs. number of $\mathrm{X}$ class $\mathrm{X}$-ray flares which appeared in the $\delta$-sunspot region is shown in Figure 2 by marks of asterisks. It is found that most of these $\delta$-sunspots have a ratio $\geq 4$. The average ratio for these 35 $\delta$-sunspots is 5.3 .

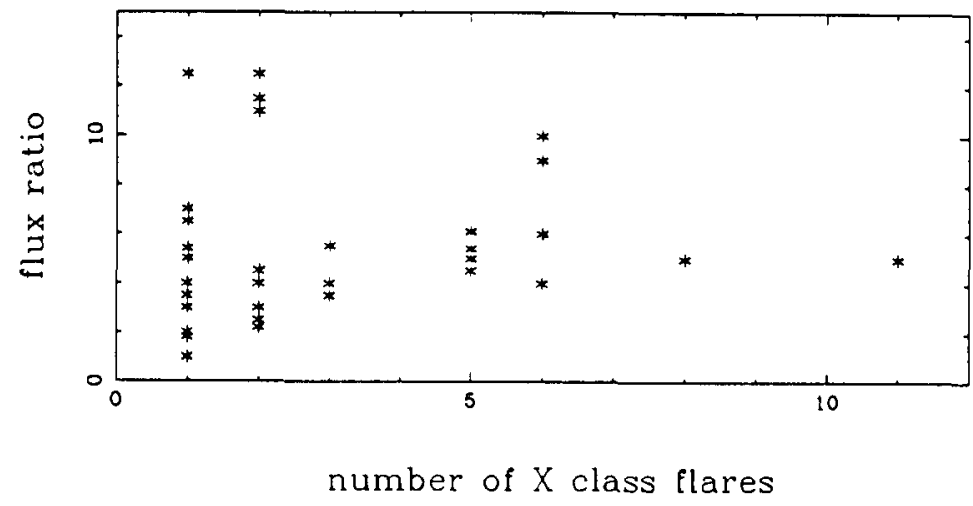

Fig.2 Scatter plot of flux ratio between majority polarity to minority one vs. number of $X$ class flares

\section{LIFETIME OF DELTA-SUNSPOTS}

The lifetime of $\delta$-sunspots varies from less than 1 day to more than 10 days. For the sample of $221 \delta$-sunspots, an average lifetime of 4.38 days is found.

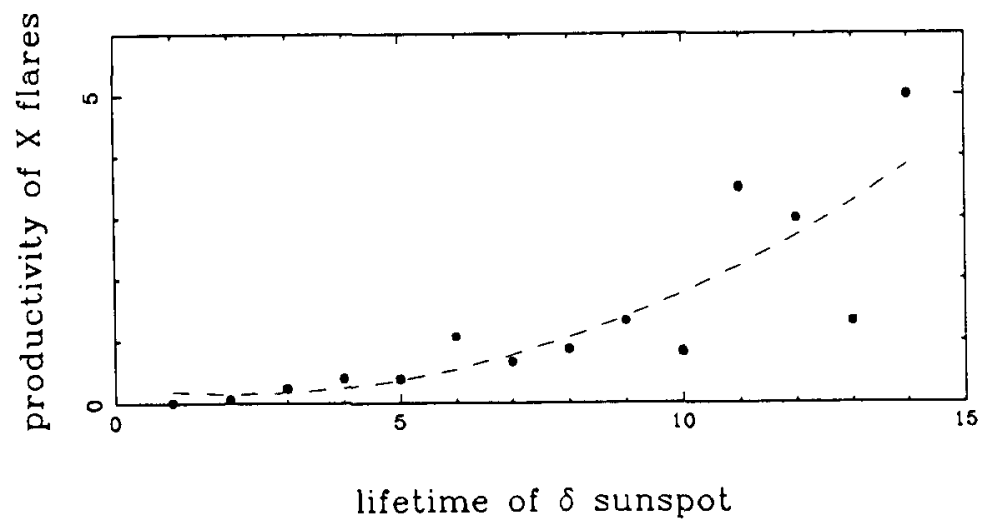

Fig.3 Scatter plot of lifetime vs. number of X class flares 
It is interesting to notice that the productivity of $\mathrm{X}$ class $\mathrm{X}$-ray flares has a rather close relationship with the lifetime of relevant $\delta$-sunspots.

For all of the $221 \delta$-sunspots, the relationship between the flare productivity, which is defined as the mean number of $\mathrm{X}$ class flares per region of given lifetime of $\delta$-sunspots and lifetime itself is illustrated. A least square fitting gives

$$
P_{x x f}=0.28-0.11 T_{\delta l f}+0.03 T_{\delta l f}^{2} \text {. }
$$

where, $P_{x x f}$ is the productivity, $T_{\delta l f}$ is the lifetime of $\delta$-sunspots.

\section{SUMMARY}

The main results of this study might be summarized as follows.

(1). Examples of the breaking up of $\delta$-sunspots have been found. The separation of $\delta$-sunspots is caused by individual proper motions of different dipoles.

(2). A highly imbalanced flux in between two opposite polarities of $\delta$ sunspots has been revealed. For 35 highly flare productive regions, the average ratio of flux from majority polarity to minority one is 5.3 .

(3). The average lifetime of $\delta$-sunspots is 4.38 days. A close relation between the productivity of $\mathrm{X}$ class flares and the lifetime of $\delta$-sunspots has been found.

\section{ACKNOWLEDGEMENT}

This work is supported by National Natural Science Foundation of China under grant 1880608 . The authors thank Huairou Stuff for providing very good magnetograms, Shahe stuff for providing sunspot data.

\section{REFERENCES}

Sheeley, N.R., Jr. 1966, Ap. J. 144, 723.

Tang, F. 1983, Sol. Phys. 89, 43.

Zirin, H. \& Liggett, M. 1987, Sol. Phys. 113, 263.

Zirin, H. 1988, Astrophysics of the Sun, Cambridge Univ. Press. 